\title{
PROCESSING BY ROBUST CEPSTRAL FRACTAL - TOPOLOGICAL METHODS OF FLOWS OF OPTICAL TEXTURE IMAGES OF THE MARTIAN SURFACE
}

\author{
Potapov A.A. ${ }^{1,2,3}$, Pakhomov A.A. ${ }^{1}$, Potapov A.A. (Jr.) ${ }^{1}$, Potapov V.A. ${ }^{1}$ \\ 1V.A. Kotelnikov IREE RAS, Moscow, Russia, potapov@cplire.ru \\ 2JNU-IREE RAS Joint Laboratory of Information Techniques and Fractal Signal Processing, \\ Jinan University, Guangzhou, China, \\ ${ }^{3}$ College of Information Science and Technology, Department of Electronic Engineering, \\ Jinan University, Guangzhou, China,
}

\begin{abstract}
The paper presents the results of applying digital image processing methods to improve their quality and intelligibility. The method is used to process images of Martian craters made by the famous American satellites Mariner and Viking. Visual analysis of processed images allows highlighting new previously unknown details. Pictures are taken from the Internet. The developed methods are suitable for processing multichannel signals with a sensor array.
\end{abstract} image

Keywords: Martian craters, multichannel signal, fractal, topology, sensor array, cepstrum, optical texture

\section{Introduction}

The problem of improving the quality of optical images of objects obtained in the presence of distortions due to the influence of the medium of propagation and radiation remains relevant in the field of astronomy, transport and aircraft control, and, naturally, in aerospace problems (in particular, target detection and selection). We also note that in recent years, interest in such a littlestudied and mysterious planet as Mars has increased noticeably in the scientific community and in the media. The possibilities of creating settlements on the surface of Mars for mining are analyzed. Similar hypotheses and programs are also discussed in the relationship of the moon. In this regard, the authors decided once again to return to the recent past, when Mars was actively explored. And using their own methods of image processing, distorted by an unknown atmosphere, the authors will try to restore in detail the most interesting parts of the planet.

\section{Mathematical statement of the problem}

When registering an optical image located at a considerable distance from the photo detector or camera, the distorting impact of the propagation medium of the received radiation takes place inevitably. Therefore, the general statement of the problem of processing or restoring an image frame is reduced to solving the convolution equation with two unknowns, after partial or complete elimination of the additive background [1-7]:

$$
i(\vec{x})=\int o(\vec{r}) h(\vec{x}-\vec{r}) d \vec{r},
$$

where $i(\vec{x})$ - registered image, $o(\vec{r})$ - initial unknown image of an unknown object, $h(\vec{x}, \vec{r})$ unknown impulse response of optical system which forms the image.

Proceeding to spatial spectra we get an expression:

$I(\vec{\omega})=O(\vec{\omega}) H(\vec{\omega})$

It is clear that without the use of additional information, this problem is unsolvable. The authors used finiteness and positivity of an unknown image $o(\vec{r})$ as additional information. Also, 
the transfer function was approximated by a Gaussian with unknown dispersion. In this case, we considered not a continuous case (2), but a discrete one - post-detector image processing. Moreover, all factors (2) are two-dimensional polynomials of finite degree. Given the features of twodimensional discrete polynomials of finite degree and the apparatus of the Lebesgue measure, we can prove that the convolution equation in the two-dimensional discrete case is almost always (in the sense of the Lebesgue measure) uniquely solved [2, 5-7].

A practical solution to this problem even with accurate knowledge of the transfer function $H(\vec{\omega})$, where $\vec{\omega}$ are the spatial frequencies, is also a difficult task and comes down to either Wiener filtering with an unknown regularizing factor or Tikhonov regularizing filters containing, in addition to the regularizing factor, several unknown stabilizing parameters [3]. Thus, there is a need to use guaranteed and monotonously converging methods for solving the problem.

The answer to this question is given by the application of set theory and, in particular, the use of projection operators on sets of functions with given properties. In solving this problem, one can use a set of compact and a set of positive functions, since it is assumed that the images have finite sizes and represent intensity distributions. Moreover, it is obvious that these sets are closed. In general terms, the procedure for solving such ill-posed inverse problems comes down to choosing an initial estimate (image), projecting it onto a set of compact functions (taking into account boundedness in linear dimensions), projecting onto a set of positive functions (zeroing negative values), and projecting onto a set of functions with given information about the Fourier spectrum.

According to a well-known theorem from functional analysis (the point theorem), if convex sets correspond to all a priori constraints, then such a procedure converges monotonously to a single true solution [4-7]. In practice, when creating algorithms for processing, due to inevitable rounding errors, determining of image sizes, the presence of noise, etc., the restoration procedure converges in a certain neighborhood, covering the selected point and lying at the intersection of convex sets. From the point of view of image restoration when applying this approach and in the presence of noise, as a result, a set of images will be obtained that is very similar to the true one, but differing from it by small intensity fluctuations that are within the noise dispersion. The external contour of the image almost does not differ from the true one, i.e. it is restored much more accurately than the distribution of image intensity.

Returning to the original problem (2) from the point of view of the described theory, it is easy to show that the set of positive and finite functions is convex [4-7]. At the same time, the set of functions (2) with a given product of spectra is a hyperbola, i.e. non-convex and non-closed set. At first glance, it seems that projection theory is not applicable in this case. However, the described difficulty is quite surmountable if we recall the methods of cepstral processing of signals and images, which come to the transition from the complex spectra themselves to their logarithms. The Fourier spectra can be written as:

$$
I(\vec{\omega})=|I(\vec{\omega})| \exp \{i \arg I(\vec{\omega})\}=|I(\vec{\omega})| \exp \left\{i \varphi_{I}(\vec{\omega})\right\} .
$$

Let us take logarithms from both sides of (2) and equate separately the real and imaginary parts:

$$
\begin{aligned}
& \operatorname{Ln}|I(\vec{\omega})|=\operatorname{Ln}|O(\vec{\omega})|+\operatorname{Ln}|H(\vec{\omega})| \\
& \varphi_{I}(\vec{\omega})=\varphi_{O}(\vec{\omega})+\varphi_{H}(\vec{\omega})
\end{aligned}
$$

Thus, it can be seen from (3) that after taking the logarithm, a set with a given product has turned into a set with a given sum. It is well known that a set with a given sum is a line, which is the limiting case of a convex set. If we introduce designation $K_{I}(\vec{\omega})=\operatorname{Ln}|I(\vec{\omega})|+i \varphi_{I}(\vec{\omega})$, where $K_{I}(\vec{\omega})$ is cepstrum of the image, then in the cepstra language, equation (2) is written as the sum:

$$
K_{I}(\vec{\omega})=K_{O}(\vec{\omega})+K_{H}(\vec{\omega}) .
$$


Thus, the possibility of converting a non-convex set (2) to a convex set (4) becomes obvious. Also, the convergence of the method for solving the convolution equation using cepstral spectra or cepstral methods becomes apparent. It is worth noting that when modeling such a procedure, the authors used modified cepstra associated with the specifics of the program (FFT); while the modified cepstrum looks like:

$$
K_{I}^{M}(\vec{\omega})=\operatorname{Ln}\{|I(\vec{\omega})|+c\} \exp i \varphi_{I}(\vec{\omega}), \quad c=0.00001,
$$

the constant $c$ is caused by the uncertainty of the logarithm value for small values of the argument.

It is easy to see that for modified cepstra the linearity of the set with a given sum is preserved, i.e. convergence of the method is guaranteed. The authors approximated the unknown transfer function $H(\vec{\omega})$ by a Gaussian, as well as its impulse response, therefore this function is real and positive and does not have a complex component, which leads to acceleration of convergence.

\section{Results Discussion}

Let us consider typical examples. In fig. 1 - fig. 4 there are the results of processing of the Martian crater Olympus Mons. The images were taken by the Mariner spacecraft in 1976. The estimated size of the crater is $25 \mathrm{~km}$ in height and $500 \mathrm{~km}$ across.
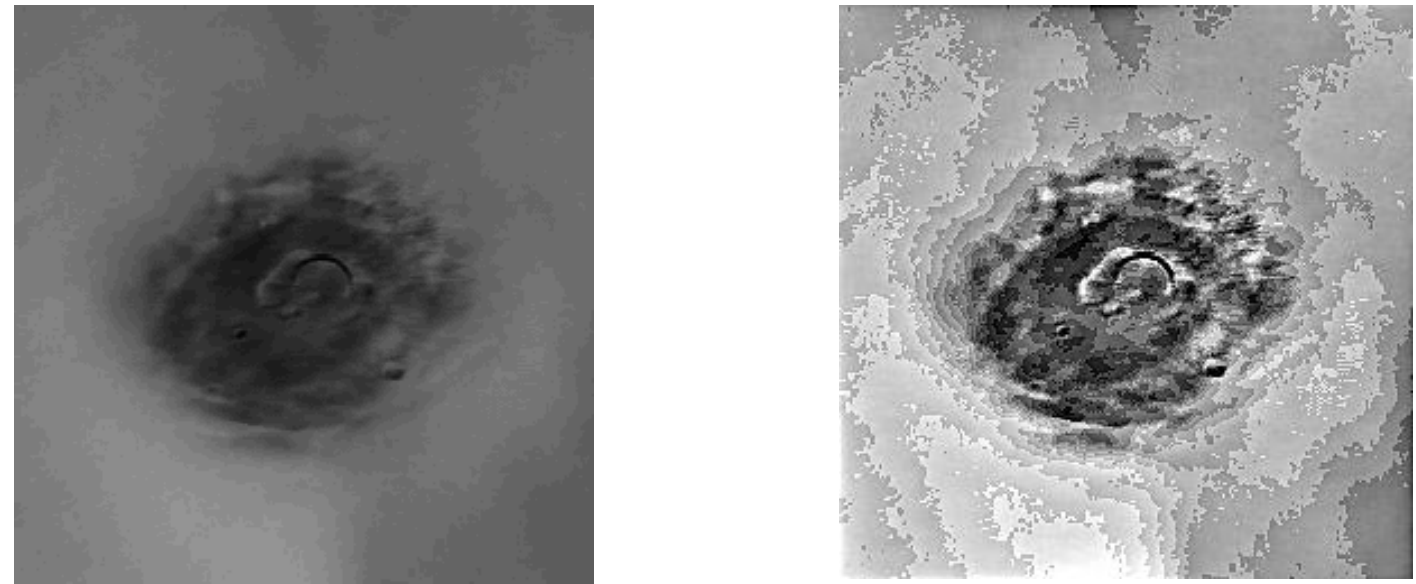

Fig.1. On the left there is the image of a fragment of the original image, on the right there is the result of processing of this fragment
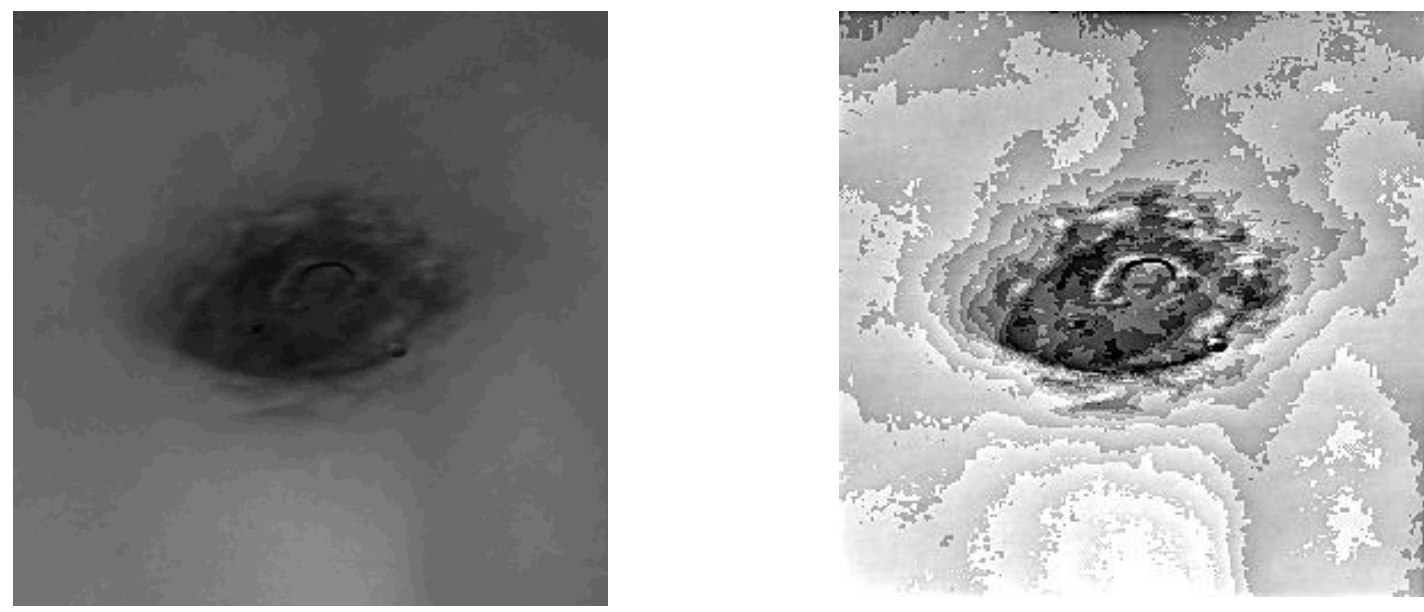

Fig.2. On the left there is the image of a fragment of the original image, on the right there is the result of processing of this fragment 

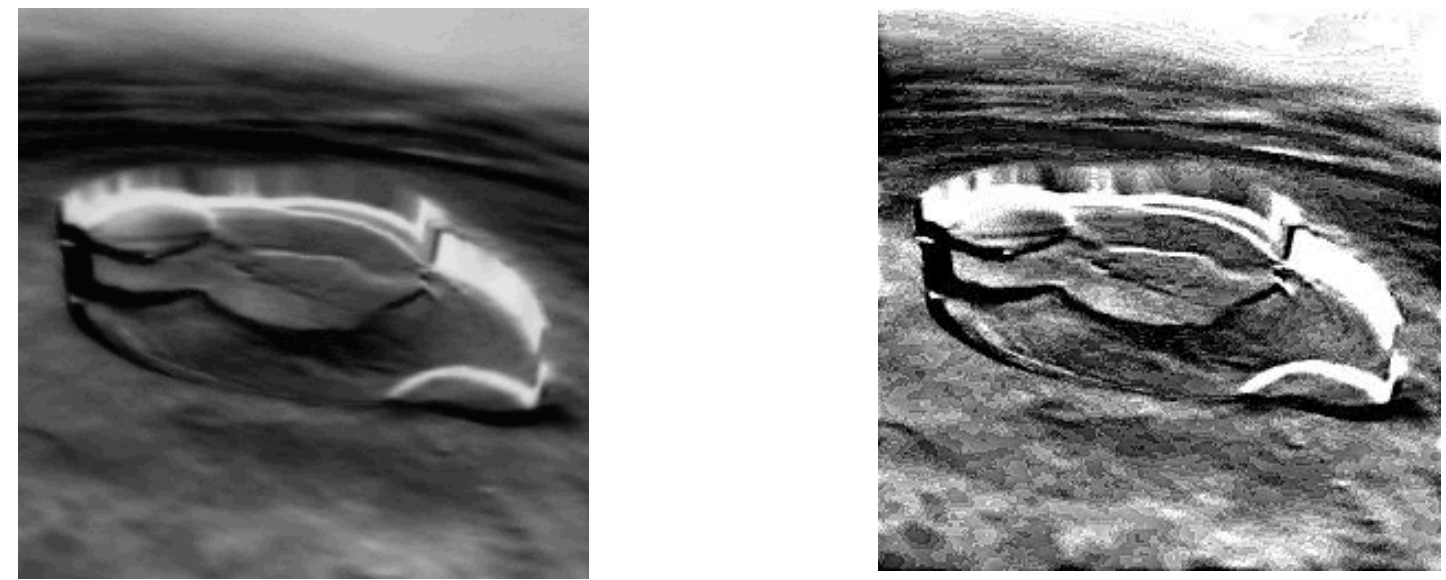

Fig. 3. On the left there is the image of a fragment of the original image, on the right there is the result of processing of this fragment
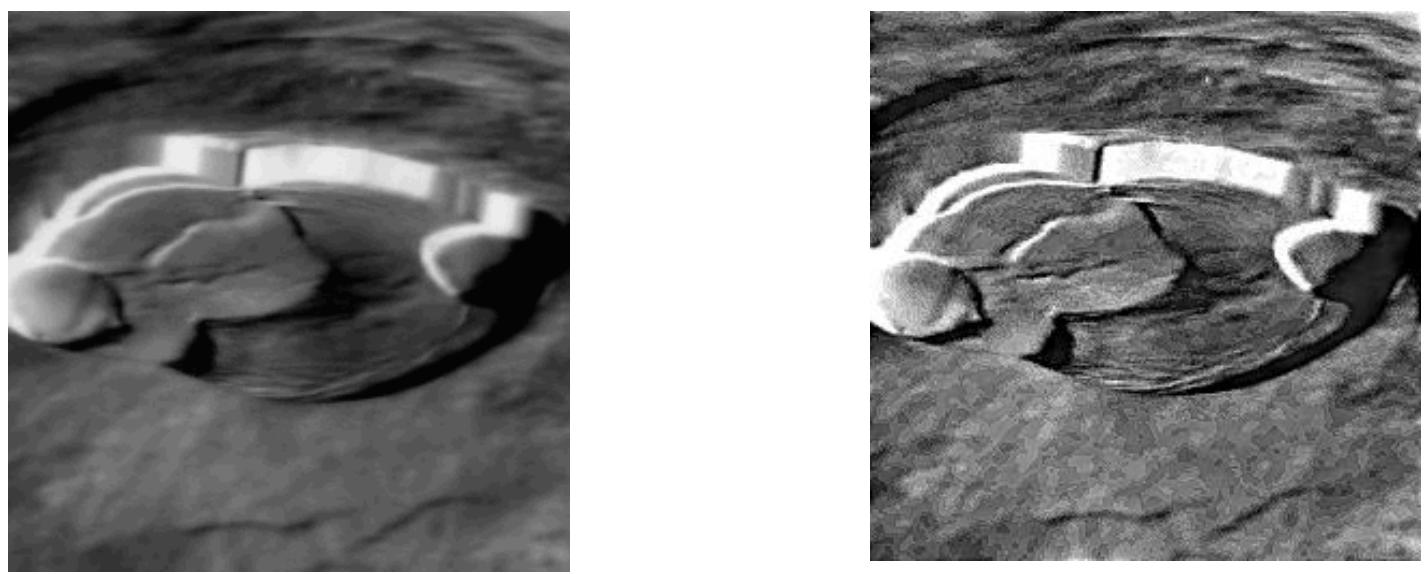

Fig. 4. On the left there is the image of a fragment of the original image, on the right there is the result of processing of this fragment

In fig. 5 - fig. 7 there are the results of processing of fragments of the Martian canyon Valles Marineris.
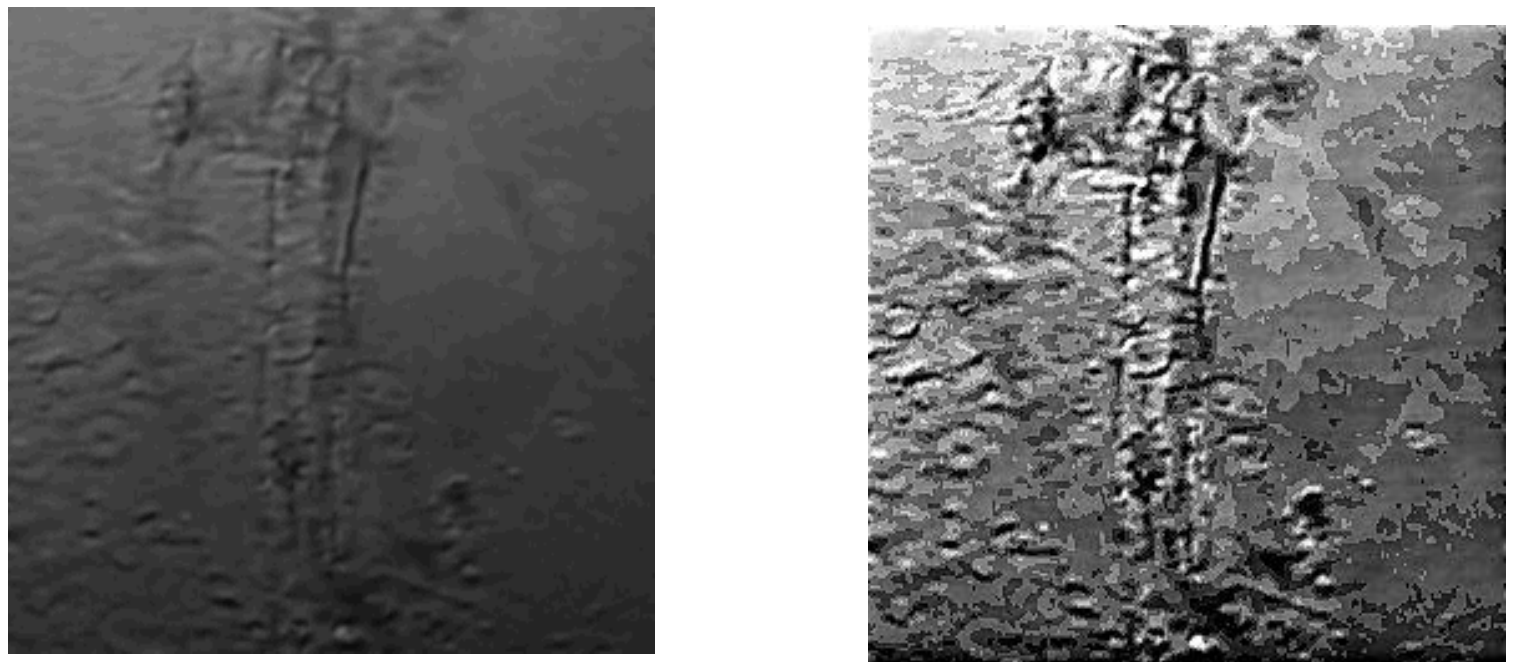

Fig.5. On the left there is the image of a fragment of the original image, on the right there is the result of processing of this fragment 

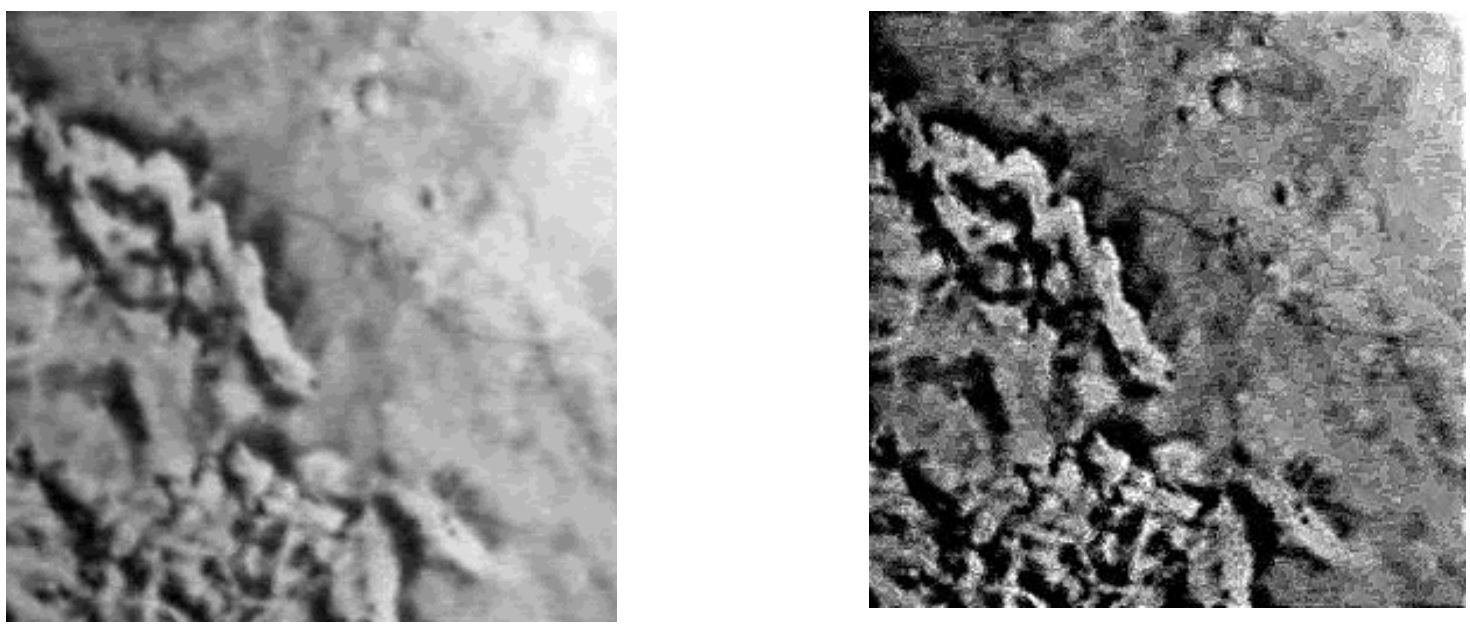

Fig.6. On the left there is the image of a fragment of the original image, on the right there is the result of processing of this fragment
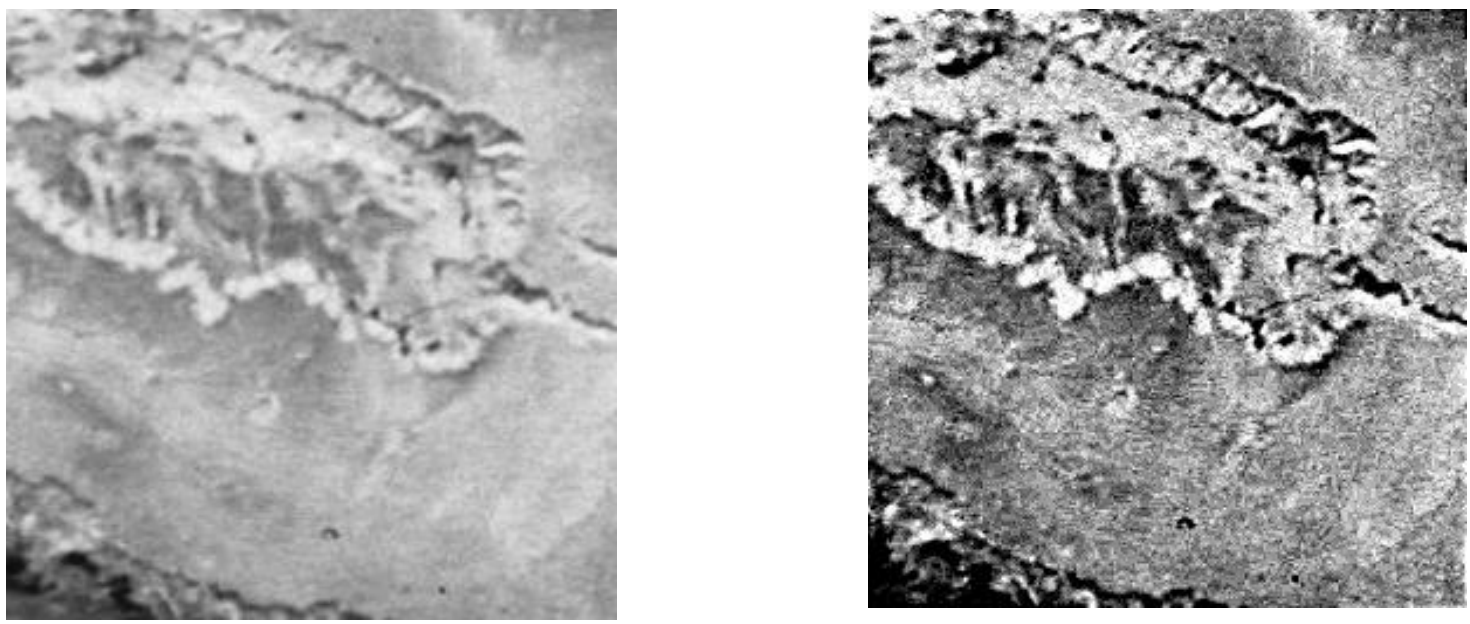

Fig.7. On the left there is the image of a fragment of the original image, on the right there is the result of processing of this fragment

Its estimated dimensions are as follows: height $10 \mathrm{~km}$, length $6000 \mathrm{~km}$, width $200 \mathrm{~km}$. The images obtained in 1976 by the Viking apparatus.

\section{Conclusion}

As conclusions, we note that the results of processing the surface of Mars presented in this paper clearly demonstrate the effectiveness of the considered approach. The methods developed by the authors are also suitable for processing of multichannel signals by a sensor matrix system and in systems of space-time adaptive processing of multidimensional signals.

\section{Acknowledgements}

The work was partially supported by the International Scientific and Technical Center (project No. 0847.2, 2000-2005, USA), the Russian Foundation for Basic Research (RFBR projects No. 05-07-90349, 07-07-07005, 07-07-12054, 07-08 -00637, 11-07-00203, 18-08-01356-a), as well as the Leading Talents Project (project No. 00201502, 2016-2020) at Jinan University (Guangzhou, China). 


\section{REFERENCES}

1 Pratt U. Digital image processing. Moscow, Mir, 1982. Book 1. - 312 p.; Book 2. - 480 p.

2 Troitskiy I.N., Ustinov N.D. Statistical theory of holography. Moscow, Radio i svyaz, 1981, 327 p. [in Russian]

3 Hayes M.H. Reconstructing of multi dimensional sequence only from amplitude or phase Fourierspectrum. IEEE Trans. Acoustic. Speech, Signal, Proc. 1982, Vol. 30, No. 3, pp. 140 - 152.

4 Vasilenko G.I., Taratorin A.M. Image restoration. Moscow, Radio i svyaz. 1986, 304 p. [in Russian]

5 Potapov A.A., Gulyaeyev Yu.V., Nikitov S.A., Pakhomov A.A., German V.A. The latest image processing methods. Moscow, FISMATLIT, 2008, 496 p. [in Russian]

6 Potapov A.A. Fractals in radio-physics and radiolocation. Moscow, Logos, 2002, 664 p. [in Russian]

7 Professor Alexander Alekseyevich Potapov. Ed. by Acad. Yu. Gulyaeyev. Moscow, 2019, 256p. [in Russian]

Article accepted for publication 22.11.2019 